\title{
Investigações no Campo da Clínica: Notas Sobre as Abordagens Analítico-Comportamental e Fenomenológica
}

\author{
Adriano Holanda \\ Jocelaine Martins da Silveira \\ Universidade Federal do Paraná
}

\begin{abstract}
RESUMO
O presente artigo se propõe a apresentar considerações sobre as possibilidades e caminhos da investigação clínica fundamentada na Fenomenologia e na Análise Comportamental. No contexto da pesquisa em clínica fenomenológica, o texto trabalha inicialmente com o direcionamento histórico das investigações para pesquisas em clínica e psicoterapia. Na atualidade, observa-se uma preocupação com os aspectos metodológicos, com as diversas possibilidades de aplicação e a abertura de novos campos, além da ênfase em aspectos epistemológicos. Por fim, discute-se a questão do que caracteriza uma investigação de cunho fenomenológico e quais seus fundamentos. A Análise Comportamental Clínica tem sua tradição fortemente inspirada em Skinner e no seu relacionamento com Ferster, cujo legado é percebido na adoção de uma terminologia comportamental na descrição de interações terapeuta/cliente, na observação de relações de consequenciação, na inclusão do comportamento do terapeuta para o exame de situações clínicas relevantes e na ênfase na idiossincrasia para prover respostas satisfatórias às questões do contexto da clínica. O principal desafio atual na pesquisa analítico-comportamental é o estabelecimento de unidades de análise que possam ser compartilhadas por diferentes pesquisadores. Alguns pontos de aproximação são discutidos: condições históricas comuns a ambos, quando reunidos sob o rótulo genérico das "psicoterapias"; a questão da ênfase idiográfica e a busca de explicações para a subjetividade.
\end{abstract}

Palavras-chave: pesquisa clínica; análise comportamental clínica; pesquisa fenomenológica.

\section{ABSTRACT \\ Investigations in the Clinical Field: \\ Notes on Behavioral Analytic and Phenomenological Approaches}

The aim of this article is to consider possibilities of investigation in the phenomenological and behavioral analysis clinical fields. In the context of clinical research in phenomenology, the present article presents initially the historical direction of clinical investigation in research and psychotherapy. Currently, there is a concern with methodological issues, the several application possibilities, and with opening up new fields, besides the emphasis on epistemological aspects. Finally, we discuss the question of what characterizes a phenomenological investigation and what its fundamentals are. The Behavior Analysis Clinic has its tradition heavily inspired by Skinner and his relationship with Ferster, whose legacy is observed in the terminology adopted to describe the therapist/client behavioral interactions, in the observation of consequences, on the inclusion of the therapist's behavior in examining clinical material, and in the emphasis on idiosyncrasy to provide satisfactory answers to questions in the clinical context. The main current challenge in behavior-analytic research is to establish units of analysis that can be shared by different researchers. Some points of possible similarities between these clinical approaches are discussed: the historical conditions common to both, when brought together under the generic label of "psychotherapy"; the question of the idiographic emphasis, and the search for explanations to subjectivity.

Keywords: clinical research; clinical behavior analysis; phenomenological research.

O campo da clínica contempla fenômenos cujas unidades de análise e de medida são um tanto quanto diversas. A pesquisa em psicologia clínica - que invariavelmente se confunde com a pesquisa em psicote- rapia - remonta a discussões sobre seus resultados, sua eficácia e efetividade (Eysenck, 1952, 1953). A pesquisa em clínica investiga os diferentes quadros nosológicos conhecidos, suas etiologias e respostas ao 
tratamento; as intervenções focadas em problemas psicológicos específicos; a comparação entre formas comprovadamente eficazes de tratamento e as variáveis da relação terapêutica. As próprias unidades de análise dos estudos variam em consistência com o viés epistemológico que fundamenta a prática clínica que está sendo examinada, aumentando assim ainda mais a diversidade de avaliações possíveis de um mesmo fenômeno, havendo soluções metodológicas sofisticadas para muitas destas investigações (Kazdin, 2003, 2003a).

Neste artigo, dedicamo-nos a comentar algumas possibilidades de investigação clínica baseadas na perspectiva fenomenológica e analítico-comportamental.

\section{Considerações sobre a Pesquisa Clínica Fenomenológica}

O contexto da produção de investigações fenomenológicas direcionadas para a clínica e a psicoterapia ainda é relativamente escasso diante tanto da complexidade do tema como do espaço ocupado pelo pensamento fenomenológico na psicologia. Como assinala Sousa (2006), o campo da pesquisa em psicoterapia surge nos primeiros anos do século passado e desde seu princípio dois caminhos - ou duas "orientações" de direção de interesse - um sendo orientado para os "resultados" e outro para o "processo". Assim, temos o seguinte quadro: a) um grupo de pesquisas se preocupa com os efeitos da psicoterapia, caracterizando-se por ser mais experimental e objetiva, com ênfase na técnica; b) enquanto um segundo grupo de pesquisas centra suas atenções sobre o processo que envolve os elementos constituintes da psicoterapia, tendo assim um enfoque subjetivo - ou melhor - intersubjetivo. A este segundo grupo de pesquisas contribui sensivelmente aqueles profissionais envolvidos com a perspectiva fenomenológica.

No esteio das pesquisas sobre a eficácia psicoterapêutica, um conjunto de profissionais - a maioria envolvida com o chamado "movimento humanista" americano, no qual despontam personalidades com Carl Rogers, Abraham Maslow, Rollo May e outros - propõem questionamentos ao primeiro campo de pesquisas, centrados exclusivamente sobre resultados objetivos que desconsideravam a dimensão subjetiva do processo clínico. O que se destaca dessa dupla consideração é um fato epistêmico, qual seja, a tradicional cisão envolvendo explicação versus compreensão, que acompanha toda a construção do saber psicológico desde seus primórdios (Sousa, 2006).

$\mathrm{O}$ primeiro conjunto de pesquisas privilegiava as técnicas e as especificidades de cada modelo psicoterápico, fazendo com que surgisse a "teoria da especificidade", defendendo a ideia de que a eficácia decorreria da técnica e do contexto específicos dos tratamentos. Como os dados empíricos não revelavam diferenças significativas entre os diversos modelos técnicos, paulatinamente foi sendo dada atenção a proposições que defendiam ênfases na "relação" como um todo - envolvendo as respectivas subjetividades e, inclusive, as técnicas. Com base nessas considerações, surge a "teoria dos fatores inespecíficos" - proposta no esteio dos questionamentos de Hans Eysenck, nos anos 50 - e, concomitantemente, os resultados apontados por Carl Rogers passam a ser considerados para além de sua ótica específica (Holanda, 1998).

O que temos constatado na atualidade é que a psicoterapia é um processo que funciona, e funciona em relação a outros contextos, tais como a fila de espera, ao placebo e ao não tratamento (Sousa, 2006). Mais recentemente percebemos um novo reavivamento do primeiro movimento, agora traduzido pela ideia dos empirically supported treatments, que visa discriminar e determinar modelos "aceitáveis" de psicoterapia para "casos" igualmente específicos. Este é o modelo que impera atualmente no contexto do seguro-saúde americano, e que vem acompanhado de um forte movimento da indústria farmacológica, principalmente no terreno da psiquiatria. $\mathrm{O}$ que se observa é que, novamente, esse modelo exclui aspectos relevantes do processo - tais como a relação em si ou as particularidades do paciente, e a dimensão subjetiva do terapeuta (Sousa, 2006).

$\mathrm{O}$ que se destaca - desde as pesquisas a partir da "teoria dos fatores inespecíficos" - é que, como assinalara Frank (1971), os fatores envolvidos num efetivo resultado de um processo psicoterapêutico passam por uma relação de confiança e carregada emocionalmente, um ambiente "facilitador" da expressão e um modelo "racional", ou seja, um conjunto coerente de teses a respeito da patogênese e da "cura". Posteriormente, e com mais detalhamentos, Altshuler (1989) propõe uma síntese entre as diversas contribuições dos modelos psicoterapêuticos, em cinco pontos: a) Uma "relação" entre terapeuta e cliente, particularmente intensa emocionalmente e caracterizada pela confiança (perceba-se que colocamos propositalmente a expressão entre aspas com vistas a destacar que o mes- 
mo significante é utilizado com diversos significados, por vezes considerados contraditórios, mas na mesma direção. Até pouco tempo atrás era comum que se considerasse como primazia de abordagens ditas fenomenológicas ou mesmo as de base analítica, a apropriação de temas tais como subjetividade ou relação sujeito-sujeito, em oposição a proposições mais objetivas); b) o apoio ou suporte ao cliente, o que gera efeitos benéficos em sua autoestima; c) a oportunidade de se expressar as emoções - tal como já havia proposto originalmente Rogers - o que leva ao encorajamento na exploração tanto dessas emoções quanto dos elementos adjacentes ao contexto, o que aproxima de noções tais como catarse ou relações contingenciais; d) um contexto "ritualístico", ou seja, cada modelo apresenta seu próprio conjunto de elementos de suporte ao processo, aumentando a confiança do cliente, e; e) uma base racional, como forma de formalizar um contexto compreensivo mínimo à sintomatologia da parte do cliente, e como uma base referencial de segurança para o terapeuta.

Todo esse movimento aproxima os diversos modelos clínicos, embora não elimine as tradicionais dicotomias ou privilégios que cada qual toma para si como forma de se apresentar na realidade. O que se observa a partir das pesquisas em clínica, num referencial fenomenológico, é um conjunto de modos de perceber o processo clínico, cujos elementos em comum são: a valorização ou privilégio do "significado" da experiência (ou o "sentido" da mesma), a ênfase no processo, a relevância de aspectos subjetivos tanto do paciente quanto do terapeuta, e o questionamento com respeito às suas bases empíricas. É sobre esses elementos que iremos tecer alguns comentários.

Observando a produção mais recente publicada em periódicos nacionais, destacam-se quatro elementos importantes: a) uma discussão em torno de modelos de pesquisa aplicados à clínica; b) uma aproximação com a discussão relativa a quadros psicopatológicos ou à psicopatologia em geral; c) um intenso debate sobre a fundamentação filosófica, tanto da prática clínica em si, quanto da própria pesquisa; e, d) a falta de uma discussão mais intensa acerca de aspectos técnicos ou metodológicos da prática clínica em si.

Principiemos pelo último ponto. Observemos que, na maioria das construções psicoterápicas, temos tido pouca ênfase no desenvolvimento e aprimoramento de aspectos técnicos ou metodológicos específicos, exceção feita aos modelos de orientação behaviorista e alguns aspectos analíticos. Na realidade, na maioria dos casos - inclusas as exceções feitas - há pouca produção crítica intrínseca, aqui compreendida como aquela que discute "desde dentro" as bases teóricas ou técnicas, sendo que o mais comum, quando surgem aspectos críticos, é a constituição de propostas supostamente "inovadoras".

Tomemos alguns exemplos ilustrativos. Podemos afirmar que todos os modelos psicoterapêuticos foram constituídos na primeira metade do século passado, senão vejamos algumas datas delimitadoras desse campo: a abordagem psicanalítica se constrói entre 1900 e a década de 30; a abordagem comportamental tem um desenvolvimento que compreende o período desde 1913 - quando da publicação do famoso texto de John Watson, Psychology as the behaviorist views it - até as reformulações propostas por B. F. Skinner, em 1953, quando da publicação de seu livro Ciência e Comportamento Humano; os modelos que se constituem como verdadeiramente psicoterapias nascem igualmente entre as décadas de 1920 a 1950, como é o caso do Psicodrama (1921, o Teatro Vienense da Espontaneidade e posteriormente, em 1931, Psicoterapia de Grupo, ambos de Moreno), a abordagem reichiana (em 1922, Reich desenvolve suas atividades de tratamento de doentes mentais com hipnose e psicoterapia, além de publicar A Função do Orgasmo em 1927), a abordagem rogeriana (em 1942, Rogers publica Psicoterapia e Consulta Psicológica e em 1951, Client-Centered Therapy), a Logoterapia (em 1946, Frankl publica A Busca do Homem por Sentido) e a Gestalt-Terapia (com a publicação, por Perls, Goodman e Hefferline, em 1951, do Gestalt-Therapy). Não poderíamos deixar de mencionar, ainda, que a abordagem junguiana se desenvolve entre 1912 - com a publicação de Transformações e Símbolos da Libido, por Jung, o que marca ainda seu rompimento com Freud e a década de 60 .

Com isto, temos um quadro singular. Temos tido um processo de continuidade e, salvo raras exceções, pouca mudança nesse quadro, onde imperam conceitos e mesmo valores desenvolvidos em épocas tardias. Em outras palavras, os modelos psicoterapêuticos atuais ainda trabalham com teses e procedimentos que pouco mudaram em cem anos, e que mesmo respondem pouco às demandas da contemporaneidade. Como assinala James Hillman, um terapeuta junguiano moderno, "já se vão cem anos de análise, as pessoas estão cada vez mais sensíveis e o mundo, cada vez pior" (Hillman \& Ventura, 1995, p. 14). 
Um exemplo ilustrativo: recentemente publicamos estudo sobre a produção acadêmica em GestaltTerapia no Brasil - aqui compreendido como o desenvolvimento de pesquisas em programas de mestrado e doutorado no país - e observamos que, num espectro relativamente pequeno (apenas 51 produções entre 1982 e 2008), a ênfase em aspectos de técnica ou procedimentos práticos não toma mais que $30 \%$ dessa produção, o que pode indicar aspectos irrefletidos na prática clínica cotidiana, aliada ao fato de termos uma cultura mais voltada à produção em livros, que perde em dinamicidade para a produção em artigos de periódicos (Holanda, 2009a).

Do ponto de vista da análise da prática clínica, a perspectiva fenomenológica subsidia as observações direcionadas para a experiência em si - enquanto ato e significado - relacionando a "vivência" dessa prática, seja do ponto de vista do cliente (como temos em Andrade, 2007), da experiência vivida que leva ao processo psicoterapêutico (Fujioka, 2009), ou das possibilidades clínicas derivadas de outras propostas de trabalho (Campos \& Cury, 2009). O que temos em comum entre essas perspectivas de pesquisa é um olhar voltado para as conexões intersubjetivas ou para as mútuas relações entre os sujeitos partícipes da relação, numa dialética entre singularidade e generalidade.

Todavia, temos observado um incremento nas discussões em torno de aspectos metodológicos referentes à investigação fenomenológica, seja aplicada à prática clínica ou a outros campos. Até o final dos anos 1990 tínhamos poucas referências acerca da pesquisa fenomenológica em solo brasileiro - o que nos remete ao intenso debate desenvolvido entre os anos 80 até o final do século passado, em torno da questão do qualitativo versus quantitativo, e ao estabelecimento de um locus assumido de pesquisa qualitativa como modelo de ciência (Holanda, 2002, 2006; Sousa, 2008) - normalmente associados a nomes tradicionais como Joel Martins, M.A.V. Bicudo, Yolanda Forghieri, William Gomes e M. Amatuzzi (Amatuzzi, 1991, 1993; Forghieri, 1993; Gomes, 1997, 1998; Martins \& Bicudo, 1989). A partir da consolidação de um espaço para as chamadas pesquisas qualitativas, a perspectiva fenomenológica passa a ganhar destaque por sua flexibilidade e aplicação a campos diversos, o que pode ser constatado com o significativo aumento da produção neste terreno (Bruns \& Holanda, 2003; Holanda, 2002, 2003a, 2003b, 2006; Giorgi, 2006; Sousa, 2006, 2008).
Como consequência desse incremento, observamos ainda que a perspectiva fenomenológica retoma outros espaços de pesquisa, alguns destes tradicionais na prática clínica, como é o caso das pesquisas associadas a quadros psicopatológicos ou relativos à psicopatologia como um todo (Moreira, 2004; Moreira \& Callou, 2006; Melo \& Moreira, 2008; Moraes, 2008; Moreira \& Telles, 2008) - o que consolida uma tradição, dado que, como assinalara Karl Jaspers, já em 1913, quando da publicação de seu "Psicopatologia Geral", a psiquiatria se constituiria na aplicação prática de um saber que, como ciência, seria a "psicopatologia" e esta deveria ser necessariamente fenomenológica (Jaspers, 1989).

Por fim, a perspectiva fenomenológica se consolida ainda como um olhar epistemológico, cujos questionamentos tocam desde um intenso debate sobre a fundamentação filosófica - tanto das práticas de pesquisa quanto das próprias práticas clínicas - o que coloca a fenomenologia no esteio das grandes correntes filosóficas que apoiam e dialogam com as ciências sociais e humanas. Especificamente sobre este ponto, temos a destacar o diálogo com os diversos campos de pesquisa em psicologia (Bruns \& Holanda, 2003) e o debate em torno das dificuldades e especificidades de aplicação desse olhar à pesquisa (Giorgi, 2006; Sousa, 2006, 2008; Castro, 2009).

Um dos temas mais discutidos atualmente diz respeito ao seguinte questionamento: o que faz uma pesquisa ser "fenomenológica"? Isto conduz a um redirecionamento das interpretações sobre a totalidade do pensamento fenomenológico, a uma releitura do que tradicionalmente se diz a respeito dela e a um novo olhar sobre os desenvolvimentos da fenomenologia contemporânea (aqui incluso as contribuições dos demais continuadores ou renovadores do pensamento husserliano), além de um novo questionamento referente ao que se compreende como pesquisa científica na contemporaneidade (como temos em González Rey, 1999, por exemplo).

Sousa (2008) ilustra essa discussão apontando consensos e dissensos na investigação fenomenológica. Por exemplo, aponta quatro pressuposições não consensuais que são: a) desenvolver pesquisa fenomenológica é fazer filosofia; b) há um número ilimitado de modos de desenhos metodológicos em fenomenologia; c) falar em método, para a fenomenologia, seria contraditório, e; d) no caso da clínica, fazer pesquisa 
fenomenológica seria o mesmo que desenvolver terapia existencial. Convém que comentemos uma a uma.

Em primeiro lugar, uma das dificuldades de se fazer pesquisa fenomenológica reside exatamente no fato que há pressupostos - como em qualquer situação de pesquisa - que devem ser respeitados, e no caso da fenomenologia, há consideráveis mal-entendidos relacionados à sua filosofia, o que nos aponta para a necessidade de uma sólida fundamentação filosófica para a tomada de posse da pesquisa fenomenológica (Holanda, 2002, 2003a, 2003b). Todavia, isto difere substancialmente da ideia de que se faz "filosofia" quando se faz pesquisa; afinal, trata-se de duas intenções diversas. Portanto, embora seja necessário um conhecimento básico de filosofia fenomenológica para se fazer pesquisa fenomenológica, os dois caminhos não podem ser confundidos.

No segundo caso, a ideia de limite está associada ao conjunto de modos de se pensar a realidade fenomênica. Sendo assim, embora tenhamos interpretações diversas da fenomenologia há, contudo, elementos que são comuns e necessários para constituir o pensamento fenomenológico como tal. Da mesma forma que não há uma psicanálise sem uma noção de inconsciente ou uma análise comportamental sem uma ideia de comportamento, não há fenomenologia sem a noção de intencionalidade.

O terceiro ponto remete a um conjunto histórico de interpretações limitadas da fenomenologia. Husserl, desde o princípio de sua obra, declara abertamente a Fenomenologia como ciência; assim, a ideia de método não apenas está contemplada aí, como é necessária para tal (Holanda, 2009b). Por fim, fazer pesquisa fenomenológica na clínica não se confunde com a prática da terapia existencial - embora os problemas e questões existenciais possam aí ser tocados - exatamente pelo fato de que se está falando, novamente, de intenções diferenciadas.

Todos esses elementos apontam para um panorama geral do que se compreende, atualmente, como pesquisa fenomenológica.

\section{Análise comportamental clínica e possibilidade de investigações de processo em Psicoterapia}

O campo que reúne práticas clínicas fundamentadas na Análise do Comportamento e de inspiração behaviorista radical tem sido designado Análise Comportamental Clínica (Dougher, 2001). Esse campo inclui estratégias terapêuticas analítico-comportamen- tais diversificadas quanto ao foco, às metas e à estrutura das sessões (Hayes, Strosahl, \& Wilson, 2003; Hayes \& Strosahl, 2005; Kohlenberg, Kanter, Kohlenberg, Folltette, \& Callaghan, 2009).

As contingências de reforço constituem a unidade capital no estudo de uma clínica fundamentada na Análise do Comportamento. De acordo com Skinner (1975),

Uma formulação das interações entre um organismo e o seu meio ambiente, para ser adequada, deve sempre especificar três coisas: 1) a ocasião na qual ocorreu a resposta, 2) a própria resposta e 3) as consequiências reforçadoras. As relações entre elas constituem as "contingências de reforço". (Skinner, 1975, p.182)

Talvez o texto que expresse mais claramente o elo do pensamento skinneriano com a pesquisa atual na Análise Comportamental Clínica seja sua publicação no Journal of the Experimental Analysis of Behavior por ocasião da morte de C. B. Ferster, em 1981.

Por cinco anos e meio Charlie Ferster e eu trabalhamos juntos na pesquisa que nós relatamos no 'Esquemas do Reforço'. Foi uma colaboração perfeita (nearperfect), sem dúvida o ponto alto na minha vida como um cientista comportamental. [...] Parte do tempo nós trabalhamos como Baconianos. Nas folhas grandes de papel, nós desenhamos tabelas mostrando esquemas programados por relógios ou contadores, com as razões ou números fixos ou variáveis, com dois ou mais esquemas em efeito simultaneamente ou em sucessão, correlacionados ou não com cores nas chaves e assim sucessivamente. Nós nos esforçamos para obter uma cobertura razoavelmente sistemática. Se nós adicionávamos DRH a um esquema de intervalo variável com bons resultados, era hora de acrescentar a ele um intervalo fixo e assim por diante. Em outras vezes nós fomos Galileicos; nós tínhamos uma teoria. A menos que nossos pombos tivessem percepção extrasensorial (uma possibilidade que nós rejeitamos), seu único contato com a programação do equipamento surgia no momento do reforçamento. Mas um certo número de estímulos poderia estar agindo naquele momento, correspondendo a leitura no velocímetro (o pássaro estava respondendo em uma dada taxa), um relógio (uma certa quantidade de tempo havia passado desde o último reforço), e um contador (um dado número de respostas havia acontecido desde o último reforço). Nós delineamos nossos experimentos para dar a estes estímulos uma oportunidade de fazer sua presença ser conhecida (Skinner, 1981, pp. 259-260)

Skinner (1981) conclui afirmando que poucas pessoas contribuíram tanto para a Análise do Comporta- 
mento quanto seu colega. Segundo Cim (2009), C. B. Ferster (1922-1981) influenciou direta e indiretamente muitas das práticas atuais na Análise Comportamental Clínica. Sua experiência no laboratório, aliada a uma visão behaviorista radical the permitiram prever o destino da pesquisa em clínica na Análise do Comportamento. Dizia ele, em 1972:

Quando clínicos e educadores rejeitam a extensão de princípios animais para o contexto terapêutico, eles muitas vezes estão reagindo implicitamente aos aspectos arbitrários dos procedimentos de laboratório em contraste com os reforços naturais do contexto clínico. A terapia comportamental, a economia de fichas, a instrução programada e outras analogias literais com o laboratório animal são somente uma forma de aplicação dos princípios comportamentais a problemas humanos. A aplicação mais geral é a de uma linguagem e conceito da natureza humana que esclarece a interação entre as condutas do indivíduo e o ambiente natural. Nesse sentido, os princípios comportamentais complementam, ao invés de substituírem abordagens clínicas empíricas para a terapia. As práticas terapêuticas são propriamente o produto de experiência acumulada e seleção natural. Uma ciência do comportamento humano é uma maneira de comunicar as partes efetivas da prática clínica e dar um traço mais refinado de experiência clínica para obter mais ganhos das interações terapêuticas. (Ferster, 1972, p. 101)

Boa parte da pesquisa que está sendo realizada atualmente sobre a interação terapeuta/cliente e os mecanismos de mudança clínica sofreu influência de Ferster $(1967,1972)$ quanto a: a) adoção de uma terminologia comportamental na descrição de interações terapeuta/cliente; b) observação de relações comportamentais na consequenciação estabelecida por terapeutas e clientes em suas interações; c) compreensão do contexto clínico como um ambiente que selecionou comportamentos típicos; d) inclusão do comportamento do terapeuta para o exame de situações clínicas relevantes, tais como a melhora do paciente; além da, e) ênfase na idiossincrasia para prover respostas satisfatórias às questões do contexto da clínica.

Segundo Callaghan, Follette, Ruckstuhl e Linnerooth (2008), os pesquisadores em psicoterapia devem cumprir as seguintes tarefas: 1) Identificar e descrever um tratamento; 2) Tornar seu mecanismo de mudança (hipotetizado) claro e mensurável; 3) Definir quais dimensões do comportamento do cliente devem mudar com aquele mecanismo; 4) Adotar um sistema de medida confiável dentro da sessão e; 5) Testar empiricamente o mecanismo com a melhora do cliente.

Callaghan, Follette, Ruckstuhl e Linnerooth (2008) recomendam também que a melhora do cliente seja registrada na relação terapêutica e que sua generalização para outros relacionamentos da vida do cliente seja demonstrada. Atualmente, há instrumentos desenvolvidos para mensuração da interação terapeuta/cliente e dos mecanismos de mudança clínica para estratégias terapêuticas focadas em relacionamentos íntimos, como costumam ser as psicoterapias (Callaghan \& Follete, 2008; Busch, Kanter, Callaghan, Baruck, Weeks, \& Berlin, 2009; Zamignani, 2008). A tecnologia recentemente desenvolvida e validada tem proporcionado mais perguntas e mais respostas sobre a relação terapêutica e a mudança clínica (Busch, Callaghan, Kanter, Baruch, \& Weeks, 2010; Donadone, 2009; Ferro-Garcia, 2008; Kanter, Landes, Busch, Rusch, Brown, Baruch, \& Holman, 2006).

Entretanto, no contexto da clínica, em razão da complexidade e amplitude do fenômeno que envolve a relação terapêutica, a unidade de análise dos estudos ainda não está claramente estabelecida e nem sempre é compartilhada entre as pesquisas. Ora opta-se pela delimitação temática das verbalizações terapeuta/cliente; ora pelo momento em que se alternam os interlocutores terapeuta e cliente. Uma tentativa de resolver o dilema do recorte do fenômeno clínico é a identificação de classes de comportamento relevantes (tanto do terapeuta, quanto do cliente) de modo que observadores treinados possam verificar sua frequência durante as sessões de terapia.

A Análise Comportamental Clínica se ressente de uma unidade de análise pouco exata. Uma unidade de análise mais precisa permitiria a consolidação dos dados, mantendo-se a mesma de um estudo para outro, de um delineamento de pesquisa para outro. Isto é, a mesma contingência relevante no contexto da clínica que fora isolada e avaliada em um estudo passado pode ser também estudada em investigações subsequentes.

A seguir, serão apresentadas algumas noções da pesquisa em Análise do Comportamento, as quais fazem supor que a missão dos estudos atuais no campo da Análise Comportamental Clínica seja a de tornar a unidade de análise mais acurada, delimitando com precisão a contingência em vigor e suas relações com a frequência do responder clinicamente relevante 
durante sessões de terapia. É possível que a contingência seja a unidade de análise fundamental, enquanto a frequência do responder constitui a unidade de medida. Mas hoje estamos limitados ao que é possível metodologicamente quando se trata do estudo de um processo de psicoterápico. Como saber se determinadas instâncias do comportamento do cliente sofrem influência dos procedimentos terapêuticos adotados? Nas pesquisas atuais, um conjunto de sessões é agrupado em fases de um delineamento experimental de caso único com replicação intra e intersujeitos, com uso de categorias para analisar a interação terapeuta/cliente. Desse modo, contingências em vigor são apenas inferidas após a análise dos dados, mas não manipuladas diretamente (Freitas, 2011; Meurer, 2011; Oshiro, 2011; Sadi, 2011; Xavier, 2011).

Ciência, na concepção de um analista comportamental é o comportamento do cientista. Johnston e Pennypacker (1993) explicam que cada etapa de um projeto de pesquisa é expresso em operantes do cientista que aumentam a probabilidade de passos subsequentes na investigação. Os passos do cientista são comportamentos de decidir que vão definindo o curso da pesquisa. Assim, a delimitação do problema da pesquisa relaciona-se com a posterior descrição de seus objetivos e, além disso, também de seu método. Cada etapa do método manterá consistência lógica e operacional com passos anteriores e subsequentes no decorrer do estudo.

De acordo com Matos (1990), a Análise Experimental do Comportamento (AEC) é o estudo do comportamento operante. Seu método é a replicação sistemática, seja ela entre arranjos de contingência, entre indivíduos de uma mesma espécie ou entre espécies. A replicação que se repete sistematicamente permite conhecer o que acontece com o comportamento, isto é, com a relação organismo/ambiente, quando arranjos e indivíduos variam. O delineamento experimental de sujeito único é o desenho por excelência da AEC. Nesse tipo de delineamento, o sujeito é seu próprio controle, em oposição aos delineamentos que recorrem à comparação entre grupos. Nesse programa de investigação, a variação é bem-vinda e move os estudos ulteriores. "(...) não acreditamos que todos os sujeitos são iguais; a variabilidade é objeto de estudo, não sinônimo de erro" (Matos, 1990, p. 586). Em tratamentos de grupo, por outro lado, qualquer variação é indesejável, grupos ideais são homogêneos.

Branch (1999) ilustra a importância do tratamento idiográfico para um analista comportamental afirman- do: "uma taxa de $2 \%$ de gravidez na população pode ter um significado importante para quem faz as políticas (segurança e pública), ela não tem significado para uma mulher individual, que nunca está $2 \%$ grávida" (p. 90). O analista do comportamento trabalha com a relação organismo/ambiente e esta é sempre idiossincrásica.

Em um delineamento experimental, uma linha de base estável - fase de linha de base - indica uma dada condição que poderá ser alterada quando uma variável for introduzida na fase de intervenção ou teste. Essa variável será removida na fase seguinte, fase de reversão à linha de base, de modo que a inclinação do responder possa ser novamente inspecionada.

O delineamento pode ser organizado interrespostas, inter-ambientes ou inter-sujeitos. Assim, por exemplo, um segundo sujeito pode permanecer em linha de base, enquanto o primeiro já está sendo submetido à fase de teste. Observa-se o que acontece na tendência do responder de ambos quando foram expostos à intervenção ou teste.

Quanto à mensuração, as medidas diretas do comportamento são sempre preferíveis. Por exemplo, opta-se por observar diretamente o comportamento de ingerir alimentos em vez de medir o peso na balança (medida indireta). As medidas indiretas podem levar a enganos como aconteceria caso a diminuição do peso observada na balança tivesse ocorrido em razão do aumento da ingestão de laxantes e de atividade física e não de mudanças no comportamento de comer.

Quanto ao modo de formular a pergunta do estudo, tanto Johnston e Pennypacker (1993), quanto Matos (1990) concordam que uma questão adequada à abordagem analítico-comportamental evita a fórmula convencional: "Qual é o efeito da variável independente (VI) sobre a variável dependente (VD)? ou ainda "A hipótese nula (H0) tal será rejeitada?". Ao invés disso, em uma formulação comportamental indaga-se quais as relações entre um evento e outro, quando uma alteração na contingência acontece. Assim, as variações são bem recebidas e há menor tendenciosidade do pesquisador na produção e interpretação dos resultados - afinal, como já foi mencionado, a ciência traduz-se pelo próprio comportamento do pesquisador. Além dessas vantagens, é possível que a formulação da pergunta nesses termos provoque um grau de serendipidade desejável.

Branch (1999), que compôs o corpo editorial do periódico The Behavior Analyst de 2004 a 2007, es- 
creveu um artigo defendendo que a inferência estatística na Análise Comportamental, mais especificamente, o uso do teste de significância seja prescindível. Segundo Branch (1999) o teste de significância estatística não fornece uma estimativa quantitativa da confiabilidade de um resultado, não estima a probabilidade de que os resultados se devam ao acaso e normalmente, não responde uma pergunta para a qual uma resposta seja desconhecida. Afinal, se o teste de significância não responde a uma pergunta importante, não fornece uma estimativa quantitativa de confiabilidade dos resultados e nem de que eles tenham ocorrido ao acaso, qual a sua utilidade?

Segundo Branch (1999), os testes de significância têm sido usados por pesquisadores e têm sido exigidos por certos periódicos com o objetivo de reduzir a responsabilidade científica. Ou seja, protegem a reputação do pesquisador, no caso de uma replicação encontrar resultados diferentes. Branch (1999) lamenta os prejuízos que isso pode causar, já que a ciência tem um papel autocorretivo imensamente apoiado na replicação. Segundo o autor, os testes também são empregados para testar uma teoria. Quando se demonstra certo efeito da hipótese alternativa e rejeita-se a hipótese nula, alguma teoria ganha apoio.

Outros analistas do comportamento adotam uma postura mais moderada em relação ao uso da inferência estatística. Ator (1999) afirma que há métodos estatísticos apropriados para sujeitos-únicos ou delineamentos de caso único. Tais métodos, segundo a autora, ajudam a avaliar tratamentos nos quais as condições de controle não são ideais, como é o caso do contexto clínico. Ator (1999) defende que a inferência estatística é apropriada quando o delineamento do estudo ou a aleatorização da amostra não são suficientes. Por outro lado, Ator (1999) considera preocupante que o experimentador opte pela inferência estatística apenas por pressões da comunidade científica. Davison (1999) defende o uso da inferência estatística quando ela facilita a visualização de generalidades e invariâncias. Crosbie (1999) preconiza a inferência estatística quando a inspeção visual é insuficiente para compreender os resultados.

Em suma, os estudos na Análise Comportamental Clínica são inspirados na tradição analítico-comportamental, que é tipicamente idiográfica em vez de nomotética. Isto implica a preferência por delineamentos nos quais o sujeito é seu próprio controle e que, frequentemente, permitem inspeção visual de gráficos e dispensam inferências estatísticas. A contingência é a unidade de análise capital nesse campo, enquanto que o comportamento, como classe operante, parece ser a unidade de medida ideal, cujas aferições são diretas, sempre que possível. Nesta tradição, o método é a replicação sistemática e a variação orienta o olhar do pesquisador, em vez de perturbá-lo.

\section{CONSIDERAÇÕES FINAIS}

$\mathrm{O}$ que se pode depreender desse debate? No que consiste a especificidade da pesquisa em clínica, a partir de abordagens supostamente tão díspares como a analítico-comportamental e fenomenológica? Ou ainda, no que consiste a possibilidade de uma interlocução entre esses dois modelos - aparentemente tão diversos e mesmo considerados, por muitos, como opostos?

Alguns elementos que se depreendem são inegáveis, do ponto de vista da análise dos procedimentos operacionais da investigação psicológica, e que merecem destaque. Imediatamente podemos assinalar um fato em comum: fazer pesquisa - seja numa perspectiva ou em outra - é trilhar a senda da ciência, mesmo que se discuta o estatuto do que se compreende por "ciência" aqui. O caráter de cientificidade encontra eco nas duas tradições, mesmo que eventualmente tenham partido - historicamente - de pontos distintos: na perspectiva analítico-comportamental sempre houve uma defesa intransigente da cientificidade (pela vertente empírico-positivista) e na perspectiva fenomenológica temos, desde o projeto husserliano, a clara proposição de uma filosofia como "ciência rigorosa".

Ao se propor, em pesquisa analítico-comportamental, a elaboração de estratégias terapêuticas, ou a identificação e descrição de um tratamento clínico, este modelo de investigação finda por encontrar os mesmos questionamentos na tradição fenomenológica. $\mathrm{Na}$ pesquisa clínica, a atenção para as dimensões de mudança envolvem tanto o lado do cliente (naquilo que é passível de ser registrado como elementos efetivos de melhora e de generalização para o contexto pós-terapia), quanto o lado do terapeuta (na descrição e identificação de modelos de ação relevantes para o processo terapêutico).

Dois outros pontos de aproximação - possivelmente polêmicos e que merecem discussão - podem ser ainda destacados entre os dois modelos de análise. Um deles diz respeito às propostas de delineamento experimental de sujeito único na Análise Comporta- 
mental Clínica, o que constitui - como defendido neste artigo - na consideração desta abordagem como idiográfica. Isto coloca a Análise Comportamental em diálogo estreito com a perspectiva fenomenológica ao considerar a singularidade do sujeito - e, portanto, seus processos de subjetivação; além disso, aponta para um antigo e esquecido debate epistemológico da psicologia moderna, que é a discussão em torno da noção de "subjetividade" e que vem sendo pouco a pouco retomada sob diversos ângulos.

O segundo ponto remete-nos aos modos de leitura do fenômeno humano em suas relações com o ambiente. A definição da psicologia como o estudo de interações (Todorov, 2007) e a noção de contingência como a relação entre eventos "ou entre comportamento e eventos ambientais na forma condicional 'se..., então..." (Todorov, 1991, p.59) remete-nos tanto à noção de intersubjetividade quanto a elaborações mais complexas da fenomenologia tardia husserliana, quando este trabalha com a ideia do Lebenswelt ou do "mundo-da-vida". As aproximações a que estamos nos remetendo refletem interpretações acerca da noção de "significado", na fenomenologia, e de "contingência", na análise comportamental.

Trata-se de uma proposição de diálogo interabordagens - e de reflexão intra-abordagens - onde possamos superar lacunas epistemológicas tradicionalmente instituídas entre modelos teóricos diversos que, apesar de partirem de aspectos distintos do mesmo fenômeno, tratam-no por caminhos paralelos. No que toca especificamente à relação organismoambiente, remetemos o leitor às elaborações propostas por Ludwig Binswanger (1977) - alicerçadas na fenomenologia de Husserl - sobre os "três mundos" de significado do homem: o Umwelt (ou mundo biológico), o Mitwelt (ou mundo social) e o Eigenwelt (o mundo-próprio ou mundo da experiência privada).

As reflexões aqui propostas caminham na direção desse diálogo epistêmico e também na elaboração de elementos de identificação para a construção de um macrocampo designado por "psicologia clínica", que ultrapasse a perspectiva psicoterápica como campo específico e recoloque a clínica na comunidade.

\section{REFERÊNCIAS}

Amatuzzi, M. M. (1991). O sentido-que-faz-sentido: Uma pesquisa fenomenológica do processo terapêutico. Psicologia: Teoria e Pesquisa, 7, 1-12.

Amatuzzi, M. M. (1993). Etapas do processo terapêutico: Um estudo exploratório. Psicologia: Teoria e Pesquisa, 9, 1-21.
Altshuler, K. Z. (1989). Will the psychotherapies yield differential results? A look at assumption in therapy trials. American Journal of Psychotherapy, 43, 310-320.

Andrade, C. C. (2007). A vivência do cliente no processo psicoterapêutico: Um estudo fenomenológico na Gestalt-Terapia. Dissertação de mestrado não-publicada, Universidade Católica de Goiânia.

Ator, N. A. (1999). Statistical inference in behavior analysis: Environmental determinants? The Behavior Analyst, 22, 93-97.

Binswanger, L. (1977). El caso de Ellen West: Estudio antropológicoclínico. Em R. May, E. Angel \& H. Ellenberger (Orgs.), Existencia (pp. 288-434). Madri: Gredos.

Branch, M. N. (1999). Statistical inference in behavior analysis: Some things significance testing does and does not do. The $\mathrm{Be}$ havior Analyst, 22, 87-92.

Bruns, M. A. T., \& A. F. Holanda (2003) (Orgs.). Psicologia e fenomenologia: Reflexões e perspectivas. Campinas: Alínea.

Busch, A. M., Callaghan, G. M., Kanter, J. W., Baruch, D. E., \& Weeks, C. (2010). The functional analytic psychotherapy rating scale: A replication and extension. Journal of Contemporary Psychotherapy, 40, 11-19.

Busch, A. M., Kanter, J. W., Callaghan, G. M., Baruck, D. E., Weeks, C. E., \& Berlin, K. S. (2009). A micro-process analysis of FAP's mechanism of change. Beha-vior Therapy, 40, 28029.

Callaghan, G. M., \& Follette, W. C. (2008). FAPRS manual: Manual for the functional analytic psychotherapy rating scale. The Behavior Analyst Today, 9, 57-97.

Callaghan, G. M., Follette, W.C., Ruckstuhl, Jr., \& Linnerooth, P. J. N. (2008). The functional analytic psychotherapy rating scale (FAPRS): A behavioral psychotherapy coding system. The Behavior Analyst Today, 9, 98-116.

Campos, A. P. S., \& Cury, V. E. (2009). Atenção psicológica clínica: Encontros terapêuticos com crianças em uma creche. Paidéia (Ribeirão Preto), 19, 115-121.

Castro, T. G. (2009). Lógica e técnica na redução fenomenológica: Da filosofia à empiria em psicologia. Dissertação de mestrado não-publicada, Universidade Federal do Rio Grande do Sul, Porto Alegre.

Cim. A. (2009). Análise comparativa entre as contribuições de $C$. B. Ferster ao campo da clínica e a psicoterapia analítica funcional (FAP). Monografia de conclusão de curso nãopublicada, Universidade Federal do Paraná, Curitiba.

Crosbie, J. (1999). Statistical inference in behavior analysis: Useful friend. The Behavior Analyst, 22, 105-108.

Davison, M. (1999). Statistical inference in behavior analysis: Having my cake and eating it? The Behavior Analyst, 22, 99103.

Donadone, J. C. (2009). Análise de contingências de orientações e auto-orientações em intervenções clínicas comportamentais. Tese de doutorado não-publicada, Universidade de São Paulo.

Dougher, M. J. (2000). Clinical behavior analysis: Theory, research and treatment. Reno: Context Press.

Eysenck, H. J. (1952). The effects of psychotherapy: An evaluation. Journal of Consulting Psychology, 16, 319-324.

Eysenck, H. J. (1953). Uses and abuses of psychology. London: Penguin Books.

Interação em Psicologia, Curitiba, 2011, 15(n. especial), p. 15-25 
Garcia, F. (2008). Recent studies in functional analytic psychotherapy. International Journal of Behavioral Consultation and Therapy, 4, 239-249.

Ferster, C. B. (1967). Arbitrary and natural reinforcement. The Psychological Record, 17, 341-347.

Ferster, C. B. (1972). Clinical reinforcement. Seminars in Psychiatry, 4, 101-111.

Forghieri, Y. C. (1993). Psicologia fenomenológica: Fundamentos, método e pesquisa. São Paulo: Pioneira.

Frank, J. (1971). Therapeutic factors in psychotherapy. American Journal of Psychotherapy, 25, 350-361.

Freitas, S. T. (2011) Efeitos de procedimentos focados na relação terapêutica sobre comportamentos geralmente descritos nos quadros de depressão. Dissertação de mestrado não-publicada, Universidade Federal do Paraná, Curitiba.

Fujioka, T. R. (2009). Solidão na relação conjugal: Um estudo fenomenológico. Dissertação de mestrado não-publicada, Universidade Católica de Goiânia.

Giorgi, A. (2006). Difficulties encountered in the application of the phenomenological method in the social sciences. Análise Psicológica, 24, 353-361.

Gomes, W. B. (1997). A entrevista fenomenológica e o estudo da experiência consciente. Psicologia USP, 8, 305-336.

Gomes, W. B. (1998). Fenomenologia e pesquisa em psicologia. Porto Alegre: Editora da UFRGS.

González Rey, F. (1999). La investigación cualitativa en psicología: Rumbos y desafíos. São Paulo: EDUC.

Hayes, S. C., \& Strosahl, K. D. (2005) (Orgs.). A practical guide to acceptance and commitment therapy. New York: SpringerVerlag.

Hayes, S. C., Strosahl, K. D., \& Wilson, K. G. (2003). Acceptance and commitment therapy: An experiential approach to behavior change. New York: Guilford Press.

Hillman, J., \& Ventura, M. (1995). Cem anos de psicoterapia... e o mundo cada vez pior. Petrópolis: Vozes.

Holanda, A. F. (1998). Diálogo e psicoterapia: Correlações entre Carl Rogers e Martin Buber. São Paulo: Lemos.

Holanda, A. (2002). O resgate da fenomenologia de Husserl e a pesquisa em psicologia. Tese de doutorado não-publicada, Pontifícia Universidade Católica de Campinas.

Holanda, A. (2003a). Fundamentação fenomenológica da pesquisa no vivido. Em I. Costa, A. F. Holanda, F. Martins, \& M. I. Tafuri (Orgs.), Ética, linguagem e sofrimento (pp. 171-183). Brasília: Abrafipp.

Holanda, A. (2003b). Pesquisa fenomenológica e psicologia eidética: Elementos para um entendimento metodológico. Em M. A. Toledo Bruns, \& A. F. Holanda (Orgs.). Psicologia e fenomenologia: Reflexões e perspectivas (pp. 35-56). Campinas: Alínea.

Holanda, A. (2006). Questões sobre pesquisa qualitativa e pesquisa fenomenológica. Análise Psicológica, 24, 363-372.

Holanda, A .F. (2009a). Gestalt-terapia e abordagem gestáltica no Brasil: Análise de mestrados e doutorados (1982-2008). Estudos e Pesquisas em Psicologia, 9, 96-121.

Holanda, A. F. (2009b). Fenomenologia e psicologia: Diálogos e interlocuções. Revista da Abordagem Gestáltica, 15, 87-92.
Jaspers, K. (1989). Psicopatologia geral (S. P. Reis, Trad.). São Paulo: Atheneu.

Johnston, J. M., \& Pennypacker, H. S. (1993). Strategies and tactics of behavioral research. Hillsdale, NJ: Lawrence Erlbaum.

Kanter, J. W., Landes, S. J., Busch, A. M., Rusch, L. C., Brown, K. R., Baruch, D. E., \& Holman, G. I. (2006). The effect of contingent reinforcement on target variables on outpatient psychotherapy for depression: A successful an unsuccessful case using functional analytic psychotyerapy. Journal of Applied Behavior Analysis, 4, 463-467.

Kazdin, A. E. (2003). Research design in clinical psychology. Needham Heights, MA: Allyn \& Bacon.

Kazdin, A. E. (2003a). Methodological issues and strategies in clinical research. Washington, DC: American Psychological Association.

Martins, J., \& Bicudo, M. A. V. (1989). A pesquisa qualitativa em psicologia: Fundamentos e recursos básicos. São Paulo: Moraes/EDUC.

Matos, M. A. (1990). Controle experimental e controle estatístico: A filosofia do caso único na pesquisa comportamental. Ciência e Cultura, 42, 585-592.

Melo, A. K. S., \& Moreira, V. (2008). Fenomenologia da queixa depressiva em adolescentes: Um estudo crítico-cultural. Aletheia, 27, 51-64.

Meurer, P. H. (2011). Efeito da apresentação de feedback no comportamento do terapeuta de evocar e responder aos comportamentos clinicamente relevantes. Dissertação de mestrado não-publicada, Universidade Federal do Paraná, Curitiba.

Moraes, C. C. (2008). Uma fenomenologia da cura espiritual: Estudo de caso na pesquisa fenomenológica. Revista da Abordagem Gestáltica, 14, 65-69.

Moreira, V., \& Callou, V. (2006). Fenomenologia da solidão na depressão. Mental, 4, 67-83.

Moreira, V., \& Telles, T. C. B. (2008). Experiências do estigma na depressão: Um estudo transcultural. Psico-USF, 13, 233-241.

Moreira, V. (2004). O método fenomenológico de Merleau-Ponty como ferramenta crítica na pesquisa em psicopatologia. Psicologia: Reflexão e Crítica, 17, 447-456.

Oshiro, C. K. B. (2011). Delineamento experimental de caso único: a psicoterapia analítica funcional com dois clientes difíceis. Tese de doutorado não-publicada, Universidade de São Paulo.

Sadi, H. M. (2011). Análise dos comportamentos de terapeuta e cliente em um caso de transtorno de personalidade borderline. Tese de doutorado não-publicada, Universidade de São Paulo.

Skinner, B. F. (1975). Contingências do reforço: Uma análise teórica (R. Moreno, Trad.). São Paulo: Abril Cultural. (Original publicado em 1969)

Skinner, B. F. (1981). Charles B. Ferster: A personal memoir. Journal of the Experimental Analysis of Behavior, 35, 259-261.

Sousa, D. (2006). Investigação em psicoterapia: Contexto, questões e controvérsias. Possíveis contributos da perspectiva fenomenológico existencial. Análise Psicológica, 24, 373-382.

Sousa, D. C. M. (2008). Dos quadros de Monet aos patos da Margarida: Divagações sobre investigação fenomenológica. Revista da Abordagem Gestáltica, 14, 21-27. 
Todorov, J. C. (1991). O conceito de contingência na psicologia experimental. Psicologia: Teoria e Pesquisa, 7, 59-70.

Todorov, J. C. (2007). A psicologia como estudo de interações. Psicologia: Teoria e Pesquisa, 23, 57-61.

Tsai, M., Kohlenberg, R. J., Kanter, J. W., Kohlenberg, B., Folltette, W. C., \& Callaghan, G. M. (2009). A guide to Functional Analytic Psychotherapy: Awareness, courage, love and behaviorism. Nova York: Springer.
Xavier, R. N. (2011). Probabilidade de transição para o estudo da modelagem em dois casos de terapia analíticocomportamental. Dissertação de mestrado não-publi-cada, Universidade de São Paulo.

Zamignani, D. R. (2008). O desenvolvimento de um sistema multidimensional para a categorização de comportamentos na interação terapêutica. Tese de doutorado não-publicada, Universidade de São Paulo.

\section{Sobre os autores:}

Jocelaine Martins da Silveira - Psicóloga, especialista em Psicoterapia na Análise do Comportamento (Universidade Estadual de Londrina), Mestre em Psicologia Experimental (Universidade de São Paulo) e Doutora em Psicologia Clínica (Universidade de São Paulo). Professora do Departamento de Psicologia da Universidade Federal do Paraná.

Adriano Holanda - Psicólogo, Mestre em Psicologia Clínica pela Universidade de Brasília, Doutor em Psicologia pela Pontifícia Universidade Católica de Campinas. Professor do Departamento de Psicologia da Universidade Federal do Paraná.

Endereço eletrônico: aholanda@yahoo.com; jocelainesilveira@ufpr.br. 